\title{
Whiskers de Fibra de Sisal Obtidos sob Diferentes Condições de Hidrólise Ácida: Efeito do Tempo e da Temperatura de Extração
}

\author{
Kelcilene B. R. Teodoro \\ Laboratório Nacional de Nanotecnologia para o Agronegócio - Embrapa - São Carlos \\ Departamento de Química - UFSCar \\ Eliangela de M. Teixeira, Ana C. Corrêa, Adriana de Campos, José M. Marconcini, Luiz H. C. Mattoso \\ Laboratório Nacional de Nanotecnologia para o Agronegócio - Embrapa - São Carlos
}

\begin{abstract}
Resumo: Neste trabalho, os efeitos de diferentes condições de tempo e temperatura usados para a preparação de whiskers de sisal foram investigados com o objetivo de se determinar a influência destes parâmetros experimentais na morfologia, cristalinidade e estabilidade térmica dos materiais preparados. A obtenção dos whiskers deu-se após o pré-branqueamento da fibra de sisal com solução alcalina de peróxido de hidrogênio. A fibra branqueada foi submetida ao processo de hidrólise com solução de ácido sulfúrico $60 \%$ (m/m) sob três diferentes condições de temperatura e tempos de extração: $45{ }^{\circ} \mathrm{C}$ e 60 minutos $\left(\mathrm{WS}_{45 \_60}\right) ; 45{ }^{\circ} \mathrm{C}$ e 75 minutos $\left(\mathrm{WS}_{45} 75\right.$ ) e $60{ }^{\circ} \mathrm{C}$ e 30 minutos $\left(\mathrm{WS}_{60}{ }_{30}\right)$. Os whiskers foram caracterizados quanto à morfologia por microscopia eletrônica de transmissão (MET), quanto à cristalinidade (DRX), carga superficial (potencial zeta), teor de enxofre (análise elementar) e quanto à estabilidade térmica por termogravimetria (TGA). Os resultados mostraram que os whiskers de sisal apresentaram comprimento e diâmetro médios e 210 nm e $5 \mathrm{~nm}$ respectivamente. Devido à alta aglomeração dos whiskers, diferenças relativas às características dimensionais não puderam ser determinadas. Os resultados obtidos revelaram uma forte dependência da cristalinidade final dos whiskers com a temperatura e tempo de extração. O uso de temperatura mais alta $\left(60^{\circ} \mathrm{C}\right)$ associado a um menor tempo de extração (30 minutos) resulta em whiskers com boa estabilidade térmica $\left(235^{\circ} \mathrm{C}\right)$, maior cristalinidade e sem o comprometimento da estrutura cristalina da celulose.
\end{abstract}

Palavras-chave: Sisal, branqueamento, whiskers de sisal.

\section{Whiskers from Sisal Fibers Obtained under Different Acid Hydrolysis Conditions: Effect of Time and Temperature of Extraction}

\begin{abstract}
In this work, the effects of different conditions of time and temperature, used for the preparation of whiskers from sisal, were investigated to determine the influence of experimental parameters on morphology, crystallinity and thermal stability of materials prepared. The whiskers were obtained after the bleaching of sisal raw fiber with a solution of hydrogen peroxide alkaline. The bleached fiber was submitted to the process of hydrolysis with sulphuric acid solution $60 \mathrm{wt}$. (\%) under three different conditions of temperature and time of extraction: $45^{\circ} \mathrm{C}$ and 60 minutes $\left(\mathrm{WS}_{45-60}\right) ; 45^{\circ} \mathrm{C}$ and 75 minutes $\left(\mathrm{WS}_{45-75}\right)$ and $60{ }^{\circ} \mathrm{C}$ and 30 minutes $\left(\mathrm{WS}_{60 \_30}\right)$. The whiskers were characterized as the morphology by transmission electron microscopy (MET), crystallinity (DRX), surface charge (zeta potential), sulfur content (by elemental analysis) and thermal stability by thermogravimetry (TGA). The sisal whiskers presented an average length and diameter of $210 \mathrm{~nm}$ and $5 \mathrm{~nm}$, respectively. Due to the high agglomeration state of whiskers, differences on dimensional features could not be determined. The results showed a strong dependence on crystallinity of whiskers with temperature and time of extraction. Hydrolysis in higher temperature $\left(60^{\circ} \mathrm{C}\right)$ and lower extraction time $(30$ minutes $)$ resulted in whiskers with good thermal stability $\left(235^{\circ} \mathrm{C}\right)$, higher crystallinity and preserving the crystalline structure of cellulose.
\end{abstract}

Keywords: Sisal, cellulose bleaching, sisal whiskers.

\section{Introdução}

O uso de fibras naturais como agente de reforço no setor plástico tem se revelado bastante promissor devido à sua larga abundância, caráter renovável e sustentabilidade ao meio ambiente além de proporcionarem materiais de alto desempenho mecânico $^{[1-3]}$.

Atualmente, diversificando-se o uso de fibras celulósicas, uma nova tendência tem-se manifestado em segmentos de pesquisas que objetivam a obtenção e uso dos chamados "whiskers" de celulose. Estes são obtidos após a hidrólise ácida da celulose, processo no qual acarreta a remoção da fase amorfa permanecendo a fase cristalina da celulose. Os whiskers têm diferentes razões de aspecto (comprimento/diâmetro (L/D)) dependendo das condições de extração e da origem da celulose ${ }^{[4]}$. Apresentam-se na forma de finas hastes (acicular) e devido à sua alta área superficial e rigidez, apresentam grande potencial como reforço em matrizes poliméricas $^{[2,5]}$.

A fibra de sisal (Agave sisalana) tem uma produção anual de 4,5 milhões de toneladas e o Brasil é o maior produtor mundial (Li et al.; Satyanarayana et al. apud Siqueira et al. $)^{[6]}$. É uma fibra muito utilizada na indústria de cordearia e no setor artesão. Das folhas do sisal retiram-se as fibras através de processos como o desfibrilamento. Devido ao seu alto conteúdo de celulose (50\%-74\%), a fibra de sisal vem sendo estudada como fonte para a obtenção de whiskers.

Os primeiros estudos envolvendo a obtenção de whiskers de sisal foram realizados por De Rodrigues et al. ${ }^{[7]}$ em 2006. Neste estudo os whiskers foram obtidos após o branqueamento da fibra de sisal empregando-se uma pré-mercerização seguida do uso de reagente clorito de sódio $\left(\mathrm{NaClO}_{2}\right)$. A hidrólise ocorreu com o uso 
de $\mathrm{H}_{2} \mathrm{SO}_{4} 65 \%(\mathrm{~m} / \mathrm{m})$ empregando-se temperatura $60{ }^{\circ} \mathrm{C}$ e tempo de extração de 15 minutos. O comprimento (L) e o diâmetro médio (D) dos whiskers foram de $250 \mathrm{~nm}$ e $4 \mathrm{~nm}$ respectivamente obtendo-se uma razão de aspecto (L/D) de 60.

Morán et al. $(2008)^{[8]}$ realizaram um complexo estudo envolvendo diferentes tipos de branqueamento da fibra de sisal. No primeiro deles, empregaram-se sucessivas etapas de branqueamento as quais foram: tratamento da fibra com $\mathrm{NaOH}$ em solução de etanol; borato de sódio alcalino, ácido nítrico e lavagem com etanol. No segundo estudo de branqueamento, as fibras foram tratadas clorito de sódio de forma similar ao proposto por De Rodriguez et al. ${ }^{[6]}$. Para a obtenção dos whiskers de sisal, as fibras pré-tratadas foram submetidas a hidrolise ácida empregando-se solução de $\mathrm{H}_{2} \mathrm{SO}_{4} 60 \%$ $(\mathrm{m} / \mathrm{m})$ e temperatura de extração a $45^{\circ} \mathrm{C}$ com exposição ao ácido por 30 minutos. O diâmetro médio encontrado foi de $30 \pm 12 \mathrm{~nm}$. Apesar do alto valor em relação aos encontrado por De Rodriguez et al. ${ }^{[7]}$, estes não são comparáveis por que foram determinados através do uso de técnicas diferentes.

De Rodriguez et al. ${ }^{[7]}$ avaliaram as dimensões por microscopia eletrônica de transmissão, enquanto Morán et al. ${ }^{[8]}$ utilizaram a técnica de microscopia de força atômica a qual, sobrestima as dimensões ${ }^{[9]}$.

Recentemente, Siqueira et al. ${ }^{[10]}$ obtiveram whiskers de sisal após o branqueamento da fibra também com clorito de sódio. A temperatura de extração empregada foi de $50{ }^{\circ} \mathrm{C}$ e o tempo de 40 minutos. Os valores de comprimento e diâmetro médios (obtidos por microscopia eletrônica de transmissão) foram respectivamente de $215 \mathrm{~nm}$ e $5 \mathrm{~nm}$, resultando em uma razão de aspecto de 43 .

Relacionando-se os trabalhos de De Rodrigues et al. ${ }^{[7]} \mathrm{e}$ Siqueira et al. ${ }^{[10]}$, observa-se que para este último, os valores dimensionais foram diferentes do que os encontrados pelos primeiros autores. Segundo Siqueira et al. ${ }^{[10]}$ estas variações foram devido à diferentes origens da fibra de sisal. Nada foi questionado sobre possíveis diferenças de dimensões, cristalinidade e estabilidade térmica devido às diferentes condições de temperatura e tempo de hidrólise empregados. O clássico trabalho de Dong et al. ${ }^{[11]}$ demonstra que os whiskers são fortemente dependentes das condições de hidrólise, as quais envolvem os parâmetros tempo e temperatura de extração.

Desta forma, neste estudo, propomos a obtenção de whiskers de sisal após o branqueamento da fibra com o uso solução de $\mathrm{NaOH} 5 \%(\mathrm{~m} / \mathrm{v})$ seguida de reação com solução alcalina de peróxido de hidrogênio o qual é um reagente não clorado e menos poluente ao ambiente. Os efeitos de diferentes condições de tempo e temperatura usados para a preparação dos whiskers foram investigados com o objetivo de determinar sua influência na morfologia, cristalinidade e estabilidade térmica dos materiais preparados.

\section{Experimental}

\section{Materiais}

As fibras de sisal foram fornecidas pela Embrapa/Algodão (PB - Brasil). Os reagentes empregados para o branqueamento das fibras foram: solução de peróxido de hidrogênio $\left(\mathrm{H}_{2} \mathrm{O}_{2}, 16 \%\right.$ (v/v), Nuclear) e solução de hidróxido de sódio $(\mathrm{NaOH}, 5 \%(\mathrm{~m} / \mathrm{v})$, Qhemis). $\mathrm{Na}$ determinação dos teores de celulose, hemicelulose e lignina empregou-se solução de ácido sulfúrico ( $72 \%$ v/v, Synth), ácido acético glacial PA (Synth), clorito de sódio $\left(\mathrm{NaClO}_{2}\right.$, J. T. Baker), metanol (Synth), solução $\mathrm{NaOH} 17,5 \%(\mathrm{~m} / \mathrm{v})$ (Qhemis). Para a extração dos whiskers empregou-se solução de ácido sulfúrico $\mathrm{H}_{2} \mathrm{SO}_{4}(60 \%$ (v/v); Synth) e para a diálise das mesmas utilizou-se membrana Sigma-Aldrich (D9402).

\section{Branqueamento das fibras de sisal}

O branqueamento das fibras de sisal deu-se em duas etapas:

i) Tratamento com $\mathrm{NaOH}$

Aproximadamente $10,0 \mathrm{~g}$ da fibra moída e peneirada (16 mesh) foram colocados em um béquer contendo $200 \mathrm{~mL}$ de solução de hidróxido de sódio $(\mathrm{NaOH} 5 \%, \mathrm{~m} / \mathrm{v})$ à temperatura de $90{ }^{\circ} \mathrm{C}$. $\mathrm{O}$ sistema permaneceu sob constante agitação por 60 minutos. Após o resfriamento procedeu-se a filtração sob vácuo. O material sólido retido foi lavado com água destilada até que se atingisse $\mathrm{pH}$ neutro. A fibra foi seca em estufa com circulação de ar a $50{ }^{\circ} \mathrm{C}$ até peso constante.

ii) Branqueamento com solução de peróxido alcalino

Cerca de $5,00 \mathrm{~g}$ da fibra de sisal previamente tratada com hidróxido de sódio (conforme descrito acima) foi colocada em uma mistura de peróxido de hidrogênio $\left(\mathrm{H}_{2} \mathrm{O}_{2} \quad 16 \%\right.$ (v/v)) e hidróxido de sódio $(\mathrm{NaOH} 5 \%(\mathrm{~m} / \mathrm{v}))$ à temperatura de $55{ }^{\circ} \mathrm{C}$. O sistema permaneceu sob constante agitação por 90 minutos. Após o resfriamento procedeu-se a filtração sob vácuo lavando-se o material com água destilada até atingir $\mathrm{pH}$ neutro. A fibra foi seca em estufa com circulação de ar a $50{ }^{\circ} \mathrm{C}$ até peso constante.

\section{Determinação dos teores de lignina, celulose e hemicelulose}

O conteúdo de lignina da fibra original e branqueada foi determinado através da reação com ácido sulfúrico de acordo com a norma TAPPI-T222 om- $88^{[12]}$. O teor de holocelulose (celulose + hemiceluloses) foi determinado segundo norma TAPPI-T19 m-54 ${ }^{[13]}$ e a hemicelulose foi removida por extração alcalina, resultando majoritariamente, somente a-celulose como fase sólida. Esta última corresponde a uma das formas polimórficas da celulose a qual apresenta a célula unitária triclínica e que é insolúvel em solução de $\mathrm{NaOH} 17,5 \%{ }^{[14]}$. O teor de hemicelulose foi obtido por subtração entre os valores de holocelulose e a-celulose.

\section{Extração dos whiskers}

A extração dos whiskers foi feita por hidrólise ácida da fibra de sisal branqueada $(5,0 \mathrm{~g})$ empregando-se solução de ácido sulfúrico $\left(100 \mathrm{~mL} \mathrm{H} \mathrm{SO}_{4} 60 \%(\mathrm{~m} / \mathrm{m})\right)$ sob agitação vigorosa e constante em três diferentes condições de temperatura e tempos de reação: $45{ }^{\circ} \mathrm{C}$ e 60 minutos $\left(\mathrm{WS}_{45 \_60}\right) ; 45{ }^{\circ} \mathrm{C}$ e 75 minutos $\left(\mathrm{WS}_{45 \_75}\right)$ e $60{ }^{\circ} \mathrm{C}$ e 30 minutos $\left(\mathrm{WS}_{60}{ }_{30}\right)$. As suspensões resultantes foram submetidas à centrifugação (8 minutos, $10.000 \mathrm{rpm})$, re-suspensas em $500 \mathrm{~mL}$ de água destilada e dialisadas em água para a remoção do excesso de ácido (pH entre 6 e 7). A suspensão foi ultrasonificada (BRANSON 450) por 5 minutos e armazenada sob refrigeração.

\section{Caracterizações das fibras de sisal (FS)}

\section{Análises por microscopia eletrônica de varredura (MEV)}

A morfologia da fibra de sisal original e branqueada foi investigada utilizando-se um microscópio Carl Zeiss DSM 940-A, à 5 kV. Uma pequena quantidade de fibra foi dispersa em água com auxílio de agitação magnética e uma gota da solução resultante foi gotejada sobre uma fita de carbono aderida ao substrato e secas a $50{ }^{\circ} \mathrm{C}$. As amostras foram recobertas com uma fina camada de ouro $( \pm 15 \mathrm{~nm})$.

\section{Análises por espectroscopia de infravermelho com transformada} de Fourier (FTIR)

Os espectros de FTIR foram obtidos empregando-se um Perkin Elmer FT-IR espectrômetro, modelo Paragon 1000 no intervalo de frequência entre 4000 e $400 . \mathrm{cm}^{-1} \mathrm{com}$ uma resolução de $2 . \mathrm{cm}^{-1}$. As fibras foram maceradas em $\mathrm{KBr}(1 \% \mathrm{~m} / \mathrm{m})$ e prensadas para obtenção das pastilhas. 


\section{Análise por difração de raio $X(D R X)$}

Os difratogramas de raio $\mathrm{X}$ das fibras, foram obtidos em um difratômetro Rigaku, operando com 50 kV, 100 mA e radiação de $\mathrm{CuKa}(1=1,5406 \AA)$. Os ensaios foram realizados a temperatura ambiente $\left(25^{\circ} \mathrm{C}\right)$ e com ângulos $2 \mathrm{q}$ entre 5 e $40^{\circ}\left(2^{\circ} / \mathrm{min}\right)$. Os índices de cristalinidade das fibras e nanofibras (Ic) foram calculados por deconvolução dos picos dos difratogramas empregando-se o software Origin 7.5, tomando-se uma função de distribuição gaussiana como formato dos picos cristalinos e amorfo. Com base nas áreas sob os picos cristalinos e amorfo, foram estimados os índices de cristalinidade, após a correção da linha base, empregandose a Equação 1.

$$
I_{c}(\%)=\left(1-\frac{A_{a}}{A_{t}}\right) \times 100
$$

onde que $A_{\mathrm{a}}$ corresponde à área da curva referente à fase amorfa e $A$ corresponde à soma das áreas de todos os picos, incluindo a curva do amorfo.

\section{Termogravimetria (TGA)}

A temperatura de degradação termooxidativa das fibras foi avaliada empregando-se a técnica de TGA utilizando-se um equipamento da TGA Q500 (TA Instruments) nas seguintes condições: atmosfera na balança: nitrogênio a $40 \mathrm{~mL} / \mathrm{min}$; atmosfera no forno: ar sintético; vazão $60 \mathrm{~mL} / \mathrm{min}$; razão de aquecimento: $10{ }^{\circ} \mathrm{C} / \mathrm{min}$; intervalo de temperatura: $25^{\circ} \mathrm{C}$ a $600{ }^{\circ} \mathrm{C}$ e porta amostra de platina.

\section{Caracterizações dos whiskers de sisal (WS)}

\section{Análise por microscopia eletrônica de transmissão (MET)}

Preparou-se uma solução diluída dos whiskers e uma gota desta suspensão foi colocada sobre suportes ultrafinos ("grides") de cobre (400 mesh, Ted Pella - $n^{\circ}$ 01822) e deixada secar a temperatura ambiente. Transcorridas 24 horas, as amostras foram coradas com solução $1,5 \%$ de acetato de uranila, por imersão dos grides por 2 minutos nesta solução de contraste. As análises foram realizadas em um equipamento Tecnai ${ }^{\mathrm{TM}} \mathrm{G} 2 \mathrm{~F} 20$. O diâmetro médio bem como o comprimento médio dos whiskers foram calculados com o auxílio do programa ImageJ e pelo menos 50 medidas foram computadas.

Análise de potencial zeta

A presença de cargas superficiais foi estimada via análise do potencial zeta a partir de alíquotas das suspensões aquosas dos whiskers cujas concentrações foram igualadas a $0,010 \%(\mathrm{~m} / \mathrm{m})$. Estas foram medidas utilizando-se um equipamento Malverne 3000 Zetasizer NanoZS, (Malverne Instruments, UK). Três medidas de cada suspensão foram realizadas.

\section{Análise elementar}

Análise elementar foi realizada com o objetivo de se verificar a presença de enxofre nas nanofibras após a extração. As medidas foram realizadas em um equipamento EAGER-200 CHNSO-CE.

\section{Termogravimetria (TGA) e Difração de raio $X(D R X)$}

Uma alíquota de cada suspensão foi seca a $35^{\circ} \mathrm{C}$ por 12 horas e analisada através da técnica de TGA e DRX nas mesmas condições descritas para as fibras de sisal.

\section{Resultados e Discussão}

\section{Caracterização das fibras de sisal}

A Figura 1 apresenta o aspecto físico da fibra de sisal original, desta após o primeiro tratamento com solução $\mathrm{NaOH} 5 \%$ e seguida do branqueamento com peróxido alcalino. Nota-se que somente após o uso do peróxido houve o descoloramento da fibra.

A morfologia da superfície longitudinal da fibra de sisal antes e após o branqueamento é mostrada na Figura 2. Observa-se que na fibra original os feixes unidos pelos componentes não fibrosos (hemiceluloses e lignina), formam uma estrutura compacta (Figura 2a). Após o branqueamento, parte destes componentes ao redor dos feixes de fibras foi removida conforme também mostram os dados da Tabela 1. Estes feixes tornam-se mais individualizados (Figura 2b) e microfibrilas puderam ser visualizadas.

Os espectros FTIR da fibra de sisal original (FS) e da fibra branqueada (FSB) são apresentados na Figura 3.

A banda em $1737 \mathrm{~cm}^{-1}$ é atribuída a grupo éster acetil e urônicos de hemiceluloses e/ou a ligações éster dos grupos carboxilas presentes nas hemiceluloses e lignina. Esta banda desapareceu com o branqueamento devido à remoção de parte das hemiceluloses e lignina. O desaparecimento da banda em $1250 \mathrm{~cm}^{-1}$ com o branqueamento foi atribuído à diminuição de grupos $\mathrm{C}-\mathrm{O}-\mathrm{C}$, indicando remoção das hemiceluloses. Adicionalmente, o desaparecimento das bandas em $1505 \mathrm{~cm}^{-1}$ (ligações $\mathrm{C}=\mathrm{C}$, incluindo os anéis aromáticos da lignina) e $1375 \mathrm{~cm}^{-1}$ (deformação axial de $\mathrm{C}-\mathrm{H}$ ) também indicam a remoção dos componentes não celulósicos ${ }^{[15]}$. A banda em $896 \mathrm{~cm}^{-1}$ na fibra branqueada é típica da estrutura da celulose ${ }^{[15]}$.

Com relação à cristalinidade das fibras, a Figura 4 apresenta os difratogramas das mesmas. Observa-se que os mesmos apresentam o pico principal em $2 \theta$ igual a $22,6^{\circ}$, característico de celulose tipo ${ }^{[16]}$. Nota-se um estreitamento deste referido pico para a amostra FSB quando comparado com o da fibra sem tratamento (FS), indicando um aumento da cristalinidade. Os índices de cristalinidade obtidos foram de $60 \%$ para FS e $64 \%$ para FSB.
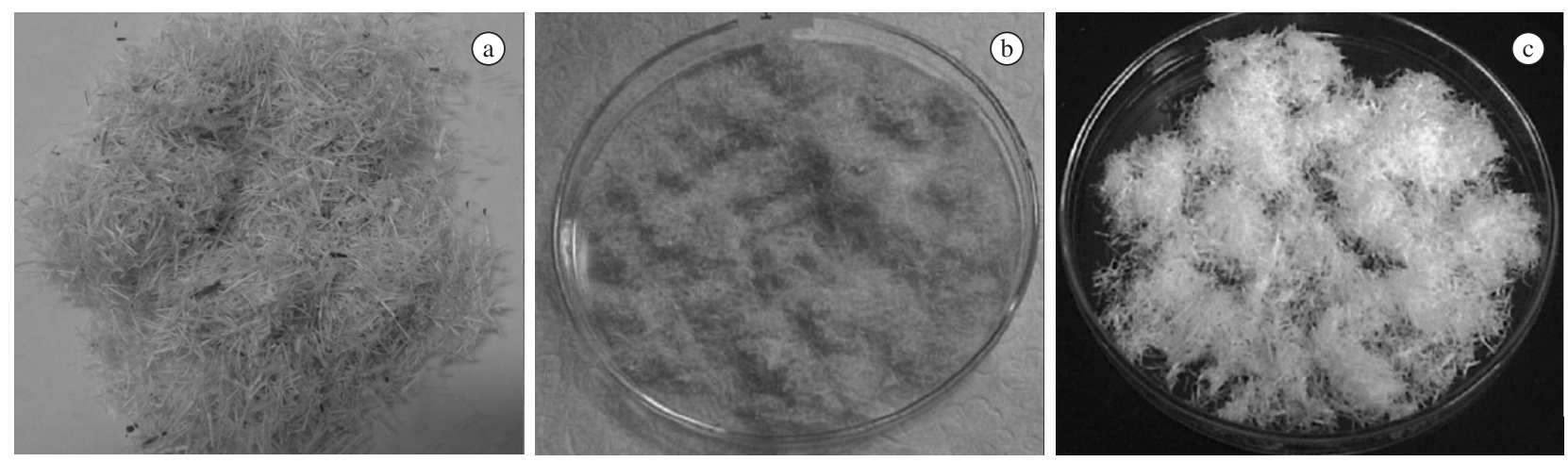

Figura 1. a) Aspecto físico da fibra de sisal original, b) após tratamento com solução $\mathrm{NaOH}$ 5\%, e c) após branqueamento com peróxido alcalino. 
Assim, os resultados das análises de FTIR, DRX e dados da Tabela 1 revelam que o tratamento com peróxido alcalino foi eficiente para a remoção de parte dos componentes não celulósicos fazendo com que a celulose ficasse mais exposta ao ataque ácido para a subseqüente etapa de extração de whiskers a partir do sisal.
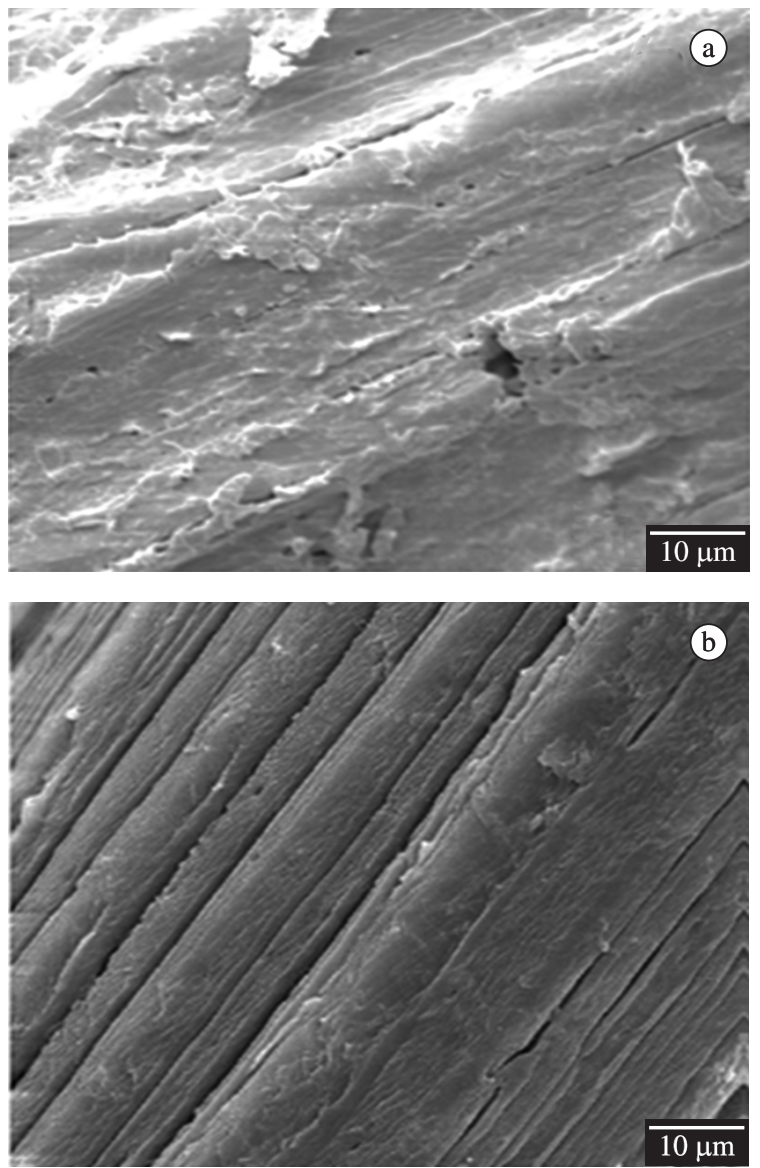

Figura 2. Micrografias de MEV da fibra de sisal: a) antes, e b) após o branqueamento com peróxido alcalino.

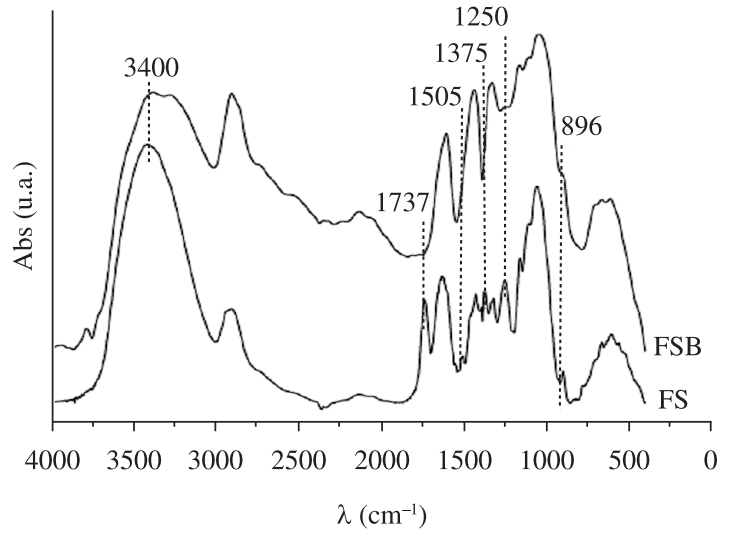

Figura 3. Espectros de FTIR da fibra de sisal original (FS) e após o branqueamento com peróxido alcalino (FSB).

\section{Caracterização dos whiskers de sisal}

A Figura 5 mostra as imagens de MET e o perfil dos difratogramas dos whiskers de sisal obtidos sob diferentes condições de tempo e temperatura. Observa-se a forma acicular típicas de whiskers de celulose $^{[2,17]}$. Os valores médios de comprimento (L) e diâmetro (D) estão dispostos na Tabela 2.

Avaliando-se as micrografias da Figura 5 e dados da Tabela 2, observa-se que as amostras $\mathrm{WS}_{45}{ }_{60}$ e WS $_{45}$ f5 foram aparentemente mais largas e compridas enquanto que os valores encontrados para a amostra $\mathrm{WS}_{60}{ }_{30}$, foram bastante similares aos já reportados na literatura ${ }^{[7,10]}$.

Segundo Dong et al. ${ }^{[1]}$ uma hidrólise incompleta pode gerar whiskers mais largos e com baixa carga superficial. A hidrólise ácida destrói as regiões amorfas das fibras e promove a enxertia dos grupos sulfatos na superfície da celulose ${ }^{[5,18]}$. Estes possibilitam a formação de uma suspensão coloidal em meio aquoso neutro (pH entre 6,0 e 7,0) cuja estabilidade da mesma é devido à repulsão eletrostática entre as cargas negativas advindas dos grupos sulfatos incorporados na superfície da celulose ${ }^{[19]}$. Assim, a presença de carga superficial na suspensão é um indicativo da presença dos grupos sulfatos na superfície da celulose ${ }^{[11]}$. Entretanto, quando se observam os valores de potencial zeta na Tabela 2 , a carga superficial de todos os whiskers foi similar entre si, indicando a efetividade da hidrólise foi praticamente igual para todas as amostras. Estes altos valores das dimensões podem ser consequência da aglomeração dos whiskers e não propriamente das condições da hidrólise. Outro resultado experimental que vem a reforçar a ocorrência da hidrólise são os obtidos por análises de DRX. Avaliando-se o perfil dos difratogramas de r-x apresentados na Figura 5 juntamente com os dados de índice de cristalinidade ( $\mathrm{I}_{\mathrm{c}}$ ) (Tabela 2) nota-se uma mudança no perfil de difração principalmente o da amostra $\mathrm{WS}_{45-75} \mathrm{e}$ a baixa cristalinidade da mesma. Este fato indica que algum nível de degradação da fase cristalina da celulose ocorreu para esta condição de extração. Para a amostra $\mathrm{WS}_{45-60}$ houve apenas um discreto aumento de $\mathrm{I}_{c}$ com relação à FSB podendo também estar ocorrendo a degradação da celulose em menor intensidade. Para a amostra $\mathrm{WS}_{60-30} \mathrm{o}$ aumento na cristalinidade relativamente à amostra FSB foi $22 \%$ indicando que nesta condição a hidrólise foi efetiva sem afetar as regiões cristalinas da celulose. Dessa forma, mesmo a uma

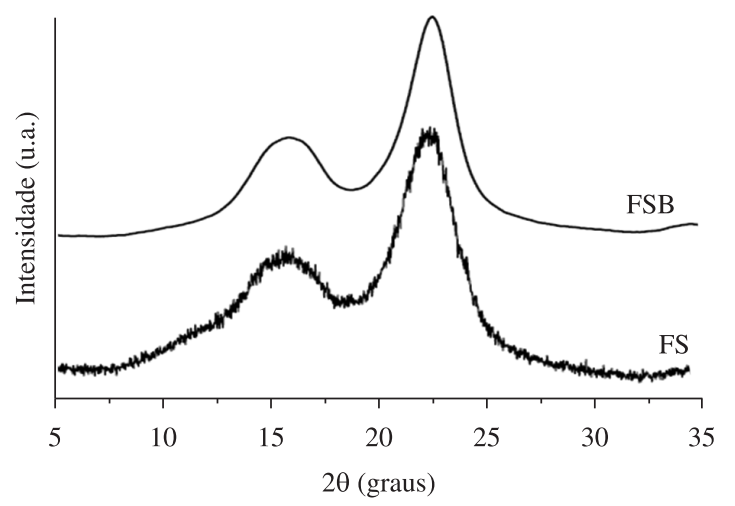

Figura 4. Difratogramas de raio X para a fibra de sisal antes (FS) e após o branqueamento (FSB).

Tabela 1. Conteúdo dos componentes da fibra de sisal antes (FS) e após o branqueamento com peróxido alcalino (FSB).

\begin{tabular}{cccccc}
\hline Amostra & $\alpha$-celulose (\%) & Hemicelulose (\%) & Lignina total (\%) & Cinzas $(\%)$ & $4 \pm 0,02$ \\
FS & $54 \pm 9$ & $25 \pm 4$ & $13 \pm 3$ & $7 \pm 1$ & $2 \pm 0,05$ \\
\hline
\end{tabular}


temperatura mais baixa $\left(45^{\circ} \mathrm{C}\right)$ mas impondo-se longos tempos de hidrólise, pode-se comprometer a estrutura cristalina da celulose. Temperaturas mais altas e tempos menores de extração favoreceram a obtenção de whiskers de sisal.

A estabilidade térmica da fibra de sisal (antes e após o branqueamento) e dos whiskers pode ser verificada através das curvas TGA/DTG como mostra a Figura 6.

Observa-se através das curvas TGA (Figura 6a) que todas as amostras apresentam uma perda inicial (7\% a $10 \%$ de massa) em torno de $50{ }^{\circ} \mathrm{C}$ a qual é devida a evaporação de água. Comparandose a FS e FSB, nota-se que devido à baixa temperatura de decomposição dos componentes não celulósicos ${ }^{[20]}$, a FS inicia sua decomposição térmica em temperatura menor que a FSB (Tabela 3).
Para esta última, o evento térmico iniciado em $280{ }^{\circ} \mathrm{C}$ corresponde à degradação das cadeias de celulose, e lignina residual. É conhecido que a degradação térmica da lignina envolve um largo intervalo de temperatura de ambiente à $900{ }^{\circ} \mathrm{C}^{[20]}$. Avaliando-se as curvas DTG (Figura 6b) para FS e FSB, observa-se um estreitamento do pico na região de $220{ }^{\circ} \mathrm{C}$ a $360{ }^{\circ} \mathrm{C}$ para a amostra FSB indicando novamente que parte dos constituintes de menor estabilidade térmica (hemiceluloses) e parte da lignina foram removidos. Apesar deste estreitamento FSB ainda apresenta um pico largo nessa região de temperatura indicando uma heterogeneidade na composição da amostra, corroborando com os dados da Tabela 1. Avaliando-se ainda os dados da Tabela 1, observa-se uma redução de $50 \%$ do teor de cinzas da fibra FSB relativamente à FS. Essa redução pode ser
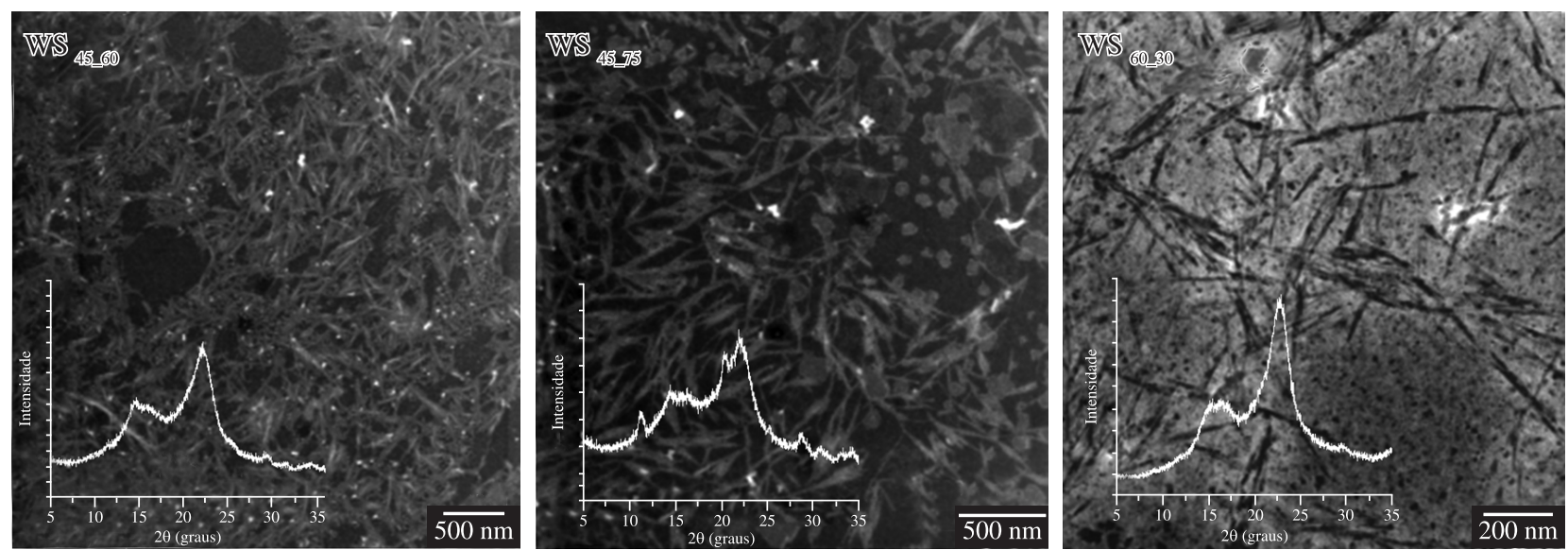

Figura 5. Micrografias de MET e difratogramas de r-x dos whiskers de sisal obtidas sob diferentes condições de temperatura e tempo, indicados na figura.

Tabela 2. Valores médios de comprimento (L), diâmetro (D), razão de aspecto, índice de cristalinidade (I) e potencial zeta das suspensões dos whiskers de sisal.

\begin{tabular}{cccccc}
\hline Amostra & L $(\mathbf{n m})$ & $\mathbf{D}(\mathbf{n m})$ & L/D & $\left(\mathbf{I}_{\mathbf{c}}\right)(\%)$ & \multicolumn{2}{c}{ Potencial zeta $(\mathbf{m V})$} \\
\hline $\mathrm{WS}_{45-60}$ & $380 \pm 75$ & $20 \pm 5$ & 19 & 66 & $-25,9 \pm 0,8$ \\
$\mathrm{WS}_{45 \_75}$ & $350 \pm 40$ & $20 \pm 5$ & 18 & 59 & $-27,0 \pm 0,8$ \\
$\mathrm{WS}_{60 \_30}$ & $210 \pm 60$ & $5 \pm 2$ & 42 & 78 & $-25,2 \pm 0,7$ \\
\hline
\end{tabular}
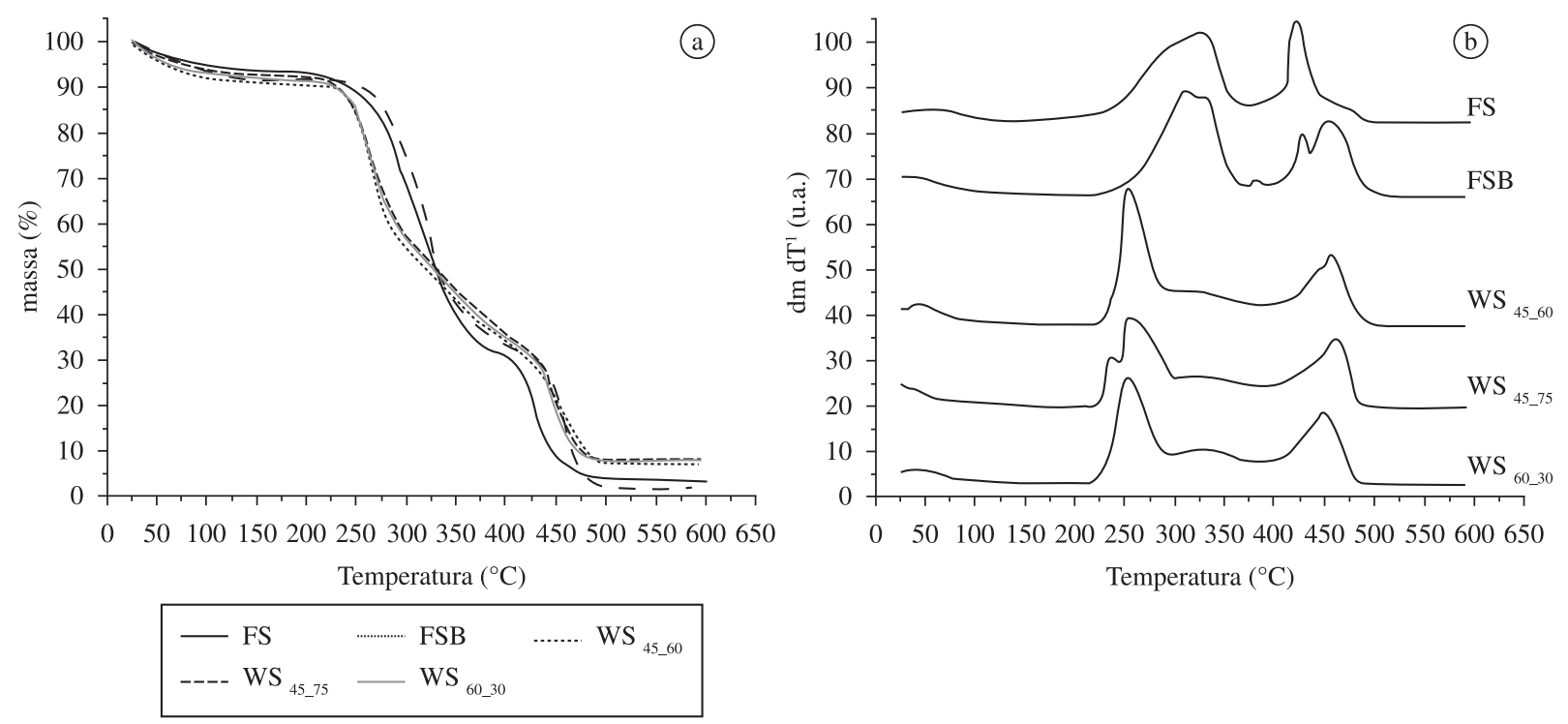

Figure 6. Curvas: a) TGA, e b) DTG da fibra de sisal original, branqueada e dos whiskers de sisal em atmosfera de ar sintético. 
Tabela 3. Temperaturas iniciais de degradação termooxidativa $\left(\mathrm{T}_{\mathrm{id}}\right)$ obtidas via termogravimetria para as fibras de sisal e whiskers e conteúdo de enxofre (S) dos whiskers obtido via análise elementar.

\begin{tabular}{ccc}
\hline Amostra & $\left(\mathbf{T}_{\text {id }}\right)\left({ }^{\circ} \mathbf{C}\right)$ & $\% \mathbf{S}$ \\
\hline FS & 250 & n.d. \\
FSB & 280 & 0,6861 \\
WS $_{45 \_60}$ & 240 & 2,5658 \\
WS $_{45 \_75}$ & 230 & 2,8280 \\
WS $_{60 \_30}$ & 235 & 2,8442 \\
\hline
\end{tabular}

n.d. = não determinado.

decorrente da remoção de parte de íons metálicos (na forma de sais dissolvidos) encontrados na fibra original ou mesmo incorporados devido às sucessivas lavagens e com contato com equipamentos utilizados, por exemplo, na sua moagem ${ }^{[21]}$.

Os whiskers resultantes apresentaram temperatura inicial de degradação térmica inferior a das fibras. Este comportamento era esperado visto que os grupos sulfatos incorporados pelo processo de extração catalisam a degradação da celulose por reações de desidratação e despolimerização da mesma ${ }^{[22,23]}$. Pelas curvas DTG dos whiskers, observa-se na amostra $\mathrm{WS}_{45-75}$ a presença nítida de ombro definido na região de $210{ }^{\circ} \mathrm{C}$ a $310{ }^{\circ} \mathrm{C}$. Esta duplicidade de picos pode estar relacionada à diferenças na efetividade de sulfonação da celulose ${ }^{[23,24]}$. Regiões mais sulfatadas da celulose degradamse em temperaturas menores enquanto que as menos acessíveis à incorporação do ácido tendem a ser mais estáveis termicamente. Dessa forma o ombro localizado na região de $210{ }^{\circ} \mathrm{C}$ na curva DTG da amostra $W_{45-75}$ refere-se a degradação da celulose cujos grupos sulfatos penetraram mais no interior das cadeias ${ }^{[23]}$. Como consequência, foram mais degradadas conforme revelou os dados de DRX (Tabela 2) e apresentaram uma $\mathrm{T}_{\text {id }}$ ligeiramente menor que a das demais amostras. A amostra $\mathrm{WS}_{45-60}$ apresenta um discreto ombro nessa mesma região de temperatura. Este comportamento sugere que esta amostra, além de possuir cadeias de celulose com grupos sulfatos incorporados superficialmente, apresenta ainda uma pequena fração de cadeias de celulose, cujos grupos sulfatos estão incorporados no interior das cadeias de celulose. Este comportamento também justifica um discreto aumento no índice de cristalinidade visto que uma parte das cadeias de celulose encontra-se degradada. $\mathrm{A}$ amostra $\mathrm{WS}_{60-30}$ apresentou um único pico na região de $210{ }^{\circ} \mathrm{C}$ a $310{ }^{\circ} \mathrm{C}$ indicando uma maior homogeneidade da sulfonação sem o comprometimento da estrutura cristalina da celulose. Comparando-se a estabilidade térmica entre os whiskers e relacionando-a ao conteúdo de enxofre incorporado (Tabela 3) nota-se que a amostra $\mathrm{WS}_{45-60}$ apresentou menor teor de enxofre entre os demais, o que explica sua estabilidade térmica ser ligeiramente maior. Quando se comparam o teor de enxofre entre as amostras $\mathrm{WS}_{45-75}$ e $\mathrm{WS}_{60-30}$ esperar-se-ia que esta última apresentasse menor estabilidade térmica. Entretanto ocorreu o inverso, confirmando o anteriormente proposto de que a estabilidade térmica não está ligada somente a quantidade de enxofre incorporada, mas também as regiões na qual os grupos sulfatos estão incorporados nas cadeias de celulose (superfície ou interior).

\section{Conclusão}

Através do uso de reagentes não clorados para a purificação da celulose contida na fibra de sisal, seguida de hidrólise ácida, foi possível a obtenção de whiskers de sisal, cujo comprimento e diâmetro médios foram de $210 \mathrm{~nm}$ e $5 \mathrm{~nm}$, respectivamente. Os resultados obtidos revelaram uma forte dependência da cristalinidade final dos whiskers com a temperatura e tempo de extração. $\mathrm{O}$ uso de temperatura mais alta $\left(60{ }^{\circ} \mathrm{C}\right)$ associado a um menor tempo de extração (30 minutos) resultou em whiskers com boa estabilidade térmica $\left(235^{\circ} \mathrm{C}\right)$ e de maior cristalinidade, sem o comprometimento da estrutura cristalina da celulose.

\section{Agradecimentos}

Os autores agradecem à FAPESP (Processos no 07/50863-4 e 08/08264-9), FINEP/MCT, CNPq e EMBRAPA.

\section{Referências Bibliográficas}

1. Samir, M. A. S. A.; Alloin, F. \& Dufresne, A. - Biomacromol, 6, p.612 (2005).

2. Hubbe, M. A.; Rojas, O. J.; Lucia, L. A. \& Sain, M. - BioResources, 3, p.929 (2008).

3. Kalia, S.; Kaith, B. S. \& Kaur, I. - Polym. Eng. Sci., 49, p.1253 (2009). http://dx.doi.org/10.1002/pen.21328

4. Dufresne, A. - J. Nanosci. Nanotechnol., 3, p.322 (2006).

5. Dufresne, A. \& Belgacem, M. N. - Polímeros, 20 (2010).

6. Siqueira, G.; Bras, J. \& Dufresne, A. - Langmuir, 26, p.402, (2009). PMid:19921797. http://dx.doi.org/10.1021/la9028595

7. De Rodriguez, N. L. G.; Thielemans, W. \& Dufresne, A. - Cellul., 13, p.261 (2006).

8. Morán, J. I.; Alvarez, V. A.; Cyras, V. P. \& Vázquez, A. - Cellul., 15, p.149 (2008).

9. Kvien, I.; Tanem, B. S. \& Oksman, K. - Biomacromol., 6, p.3160 (2005). PMid:16283741. http://dx.doi.org/10.1021/bm050479t

10. Siqueira, G.; Bras, J. \& Dufresne, A. - Biomacromol., 10, p.425 (2009). http://dx.doi.org/10.1021/bm801193d

11. Dong, X. M.; Revol, J-F. \& Gray, D. G. - Cellul., 5, p.19 (1998). http:// dx.doi.org/10.1023/A:1009260511939

12. TAPPI Standard. - "Method T222 om-88", TAPPI Test Methods (1999).

13. TAPPI Standard. - "Method T19m-54", TAPPI Test Methods (1991).

14. Rondeau-Mouro, C.; Bouchet, B.; Pontoire, B.; Robert, P.; Mazoyer, J. \& Buléon, A. - Carbohyd. Polym., 53, p.241 (2003). http://dx.doi. org/10.1016/S0144-8617(03)00069-9

15. Alemdar, A. \& Sain M. - Bioresour. Technol., 99, p.1664, (2008). PMid:17566731. http://dx.doi.org/10.1016/j.biortech.2007.04.029

16. Klemm, D.; Heublein, B.; Fink, H. P. \& Bohn, A. - Angew. Chem. Int. Ed., 44, p.2, (2005).

17. Gardner, D. J.; Oporto, G. S.; Mills, R. \& Samir, M. A. S. A. - J. Adhesion Sci. Technol., 22, p.545, (2008). http://dx.doi. org/10.1163/156856108X295509

18. Lima, M. M. S. \& Borsali, R. - Langmuir, 18, p.992, (2002). http:// dx.doi.org/10.1021/la0105127

19. Lima, M. M. S. \& Borsali, R. - Macromol. Rapid Commun., 25, p.771, (2004).

20. Yang, H.; Yan, R.; Chen, H.; Lee, D. H. \& Zheng, C. - Fuel, 86, p.1781, (2007). http://dx.doi.org/10.1016/j.fuel.2006.12.013

21. Brasileiro, L. B.; Colodete, J. L.\& Piló-Veloso, D. - Quím. Nova, 24, p.819, (2001). http://dx.doi.org/10.1590/S0100-40422001000600020

22. Roman, M. \& Winter, W. T. - Biomacromol., 5, p.1671, (2004). http:// dx.doi.org/10.1021/bm034519+

23. Li, R.; Fei, J.; Cai, Y.; Li, Y.; Feng, J. \& Yao, J. - Carbohyd. Polym., 76, p.94 (2009). http://dx.doi.org/10.1016/j.carbpol.2008.09.034

24. Wang, N.; Ding, E. \& Cheng, R. - Polym., 48, p.3486, (2007). http:// dx.doi.org/10.1016/j.polymer.2007.03.062

Enviado: $27 / 04 / 10$

Reenviado: $17 / 12 / 10$

Aceito: $24 / 02 / 11$

DOI: 10.1590/S0104-14282011005000048 